\title{
Diacronie
}

Studi di Storia Contemporanea

$N^{\circ} 24,4 \mid 2015$

Le dittature militari: fisionomia ed eredità politica

\section{Il Memoriale dell'Amnistia politica brasiliana: contesa simbolica e legittimità nella città delle "libertà"}

\section{Priscila Cabral Almeida}

Traduttore: Jacopo Bassi

\section{(2) OpenEdition Journals}

Edizione digitale

URL: http://journals.openedition.org/diacronie/3698

DOI: $10.4000 /$ diacronie.3698

ISSN: 2038-0925

Editore

Association culturelle Diacronie

Notizia bibliografica digitale

Priscila Cabral Almeida, « II Memoriale dell'Amnistia politica brasiliana: contesa simbolica e legittimità nella città delle "libertà" », Diacronie [Online], N 24, 4 | 2015, documento 7, Messo online il 29 décembre 2015, consultato il 02 mai 2019. URL : http://journals.openedition.org/diacronie/3698 ; DOI : 10.4000/ diacronie.3698 


\section{Diacronie}

N. 24 | 4|2015 Le dittature militari: fisionomia ed eredità politica

\section{0/}

\section{Il Memoriale dell'Amnistia politica brasiliana: contesa simbolica e legittimità nella città delle "libertà"}

Priscila CABRAL ALMEIDA *

traduzione a cura di Jacopo BASSI

L'obiettivo di questo articolo è analizzare il discorso politico sorto intorno alla realizzazione, a Belo Horizonte, nello Stato di Minas Gerais, del Memoriale dell'Amnistia politica brasiliana. Prescindendo dal fatto che i lavori non si sono ancora conclusi, si tratta della prima iniziativa di rilievo in termini di politiche patrimoniali da parte dello Stato brasiliano che dimostra un cambiamento di posizione nell'atteggiamento del governo federale, nell'intento di risarcire simbolicamente le vittime dei crimini perpetrati dal regime militare (1964-1985). In considerazione del fatto che esiste un'intricata rete di significati intorno allo spazio e alla memoria, così come ai discorsi politici e alle appropriazioni simboliche che circondano la costruzione del memoriale, desideriamo prendere in analisi i discorsi e i miti fondatori mineiros impiegati per l'edificio di Belo Horizonte, pensato e progettato come sintesi dell'ideale repubblicano brasiliano. Desideriamo quindi così sollevare alcune questioni e analizzare le narrazioni che riguardano l'appropriazione politica di alcuni valori, come la democrazia e la libertà, nell'ambito della cosiddetta "giustizia di transizione". 
«Joaquim José da Silva Xavier

Era il nome di Tiradentes

Si sacrificò per la nostra libertà

Questo grande eroe

Deve essere ricordato per sempre»

Mano Décio da Viola, Estanisláu Silva, Penteado

«Se molto vale ciò è stato fatto,

Più vale ciò che deve essere fatto»

Milton Nascimento, Fernando Brant

\section{Introduzione}

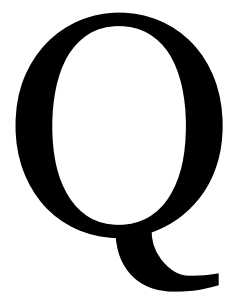

uesto articolo ha come obiettivo quello di analizzare il discorso politico inerente la realizzazione del Memoriale dell'Amnistia politica brasiliana a Belo Horizonte, nello Stato del Minas Gerais. A prescindere dal fatto che la sua edificazione non sia stata terminata, si tratta della prima iniziativa rilevante in termini di politiche patrimoniali da parte dello Stato brasiliano, che segnala un cambiamento di posizione nell'atteggiamento del governo federale frutto dell'intento di risarcire simbolicamente le vittime dei crimini perpetrati dal regime militare (1964-1985), oggi considerato dalla legislazione internazionale come crimine contro l'umanità.

La mancanza di consenso riguardo alla Legge di amnistia promulgata il 28 agosto 1979 suscita interrogativi, come vedremo successivamente, circa l'ambiguità sui possibili effetti simbolici del memoriale e, al contempo, sulle rappresentazioni che si intende mettere in circolazione nella narrazione politica nazionale.

Considerando l'esistenza di un'intricata rete di significati intorno allo spazio della memoria, così come i discorsi politici e le appropriazioni simboliche che stanno attorno alla costruzione del memoriale, abbiamo scelto di analizzare i discorsi e i miti fondatori mineiros impiegati per l'edificio di Belo Horizonte, pensato e progettato come sintesi dell'ideale repubblicano brasiliano. In questo modo intendiamo sollevare alcune questioni e analizzare le narrazioni che riguardano l'appropriazione politica di alcuni valori, come democrazia e libertà, nell'ambito di quella che viene chiamata giustizia di transizione ${ }^{1}$.

\footnotetext{
${ }^{1}$ La giustizia di transizione è un procedimento legittimato dalle politiche internazionali che interessa i paesi che hanno conosciuto un regime di eccezione e che si trovano in un processo di ridemocratizzazione e consolidamento delle istituzioni democratiche. I suoi quattro assi fondamentali sono: «(I) il risarcimento; (II) la divulgazione della verità e la costruzione della memoria; (III) la regolarizzazione della giustizia e il ristabilimento dell'uguaglianza della giustizia; (IV) la riforma delle istituzioni responsabili di violazioni dei diritti umani». ABRÃO,
} 
Per la realizzazione dell'obiettivo che abbiamo definito in precedenza, analizzeremo la letteratura consacrata ad alcuni temi, tra cui l'amnistia, la giustizia di transizione, la città e il suo patrimonio. Nel prossimo paragrafo discuteremo il progetto del Memoriale dell'Amnistia in costruzione nella città di Belo Horizonte.

\section{Il Memoriale dell'Amnistia brasiliana: un luogo della memoria della dittatura}

Nel 2009 la «Revista Anistia Política e Justiça de Transição» annunciava che il Memoriale dell'Amnistia politica brasiliana sarebbe divenuto realtà. Grazie a investimenti federali del valore di 25,6 milioni di reais, il progetto del Ministero della Giustizia in collaborazione con l'Universidade Federal de Minas Gerais (UFMG), la Prefettura di Belo Horizonte, la Segreteria del Patrimonio dell'Unione e sostenuto dalla Caixa Econômica Federal, sarebbe stato concepito per essere realizzato nello spazio occupato dal corpo principale del vecchio campus della Facoltà di Filosofia e Scienze sociali (Fafich) e dal Teatro Universitario (TU), nel quartiere centrale di Santo Antônio, a Belo Horizonte.

La concessione di questo spazio fu giustificata dalla necessità di rendere disponibili e salvaguardare i quasi 70.000 procedimenti giudiziari trattati dalla Commissione di Amnistia del Ministero di Giustizia nei confronti dei perseguitati politici dal regime militare (1964-1985), rinnovando così l'impegno dello Stato brasiliano «a compensare il danno causato a migliaia di persone da un uso discrezionale del potere», riaffermando la volontà di correggere gli errori del passato attraverso principi di accountability $^{2}$ che rinforzino la fiducia nei principi democratici3.

Paulo, TORELLY, Marcelo D., «Justiça de Transição no Brasil: a dimensão da reparação», in Revista Anistia Política e Justiça de Transição/Ministério da Justiça, 3, 1/2010, pp. 108-139, p. 110.

${ }^{2}$ Mezarobba utilizza l'espressione "principi di accountability» per designare il carattere dei più recenti sviluppi del procedimento di amnistia in Brasile. Con la creazione della Comissão de Mortos e Desaparecidos (1995) e della Comissão de Anistia (2001) - prodotti del processo di lungo periodo dell'amnistia - lo Stato brasiliano riconosce la sua responsabilità nelle gravi violazioni dei diritti attraverso le riparazioni economiche nei confronti delle vittime della dittatura militare. Tuttavia l'autore, ritiene che, al 2003, «la traiettoria tracciata dallo Stato non lascia dubbi circa il fatto che l'investimento principale sia stato fatto nel campo della giustizia amministrativa, specialmente in quella forma compensativa volta alla compensazione economica», senza «impegno a cercare la verità, o tantomeno una punizione». MEZAROBBA, Glenda,Um acerto de contas com o futuro: a anistia e suas consequências - um estudo do caso brasileiro, Tesi in Scienze politiche, Universidade de São Paulo, São Paulo, 2003, p. 146. Nella nostra valutazione dello scenario transizionale attuale, consideriamo la promozione di progetti di valore simbolico diretti dalla Comissão de Anistia - ad esempio la costruzione del Memoriale dell'Amnistia e la raccolta di testimonianze di amnistiati da parte del progetto Marcas da 
La previsione iniziale dell'inaugurazione dello spazio era per il primo aprile 2014, occasione nella quale sarebbero stati commemorati i cinquant'anni dal golpe civilemilitare. Con lo spostamento della consegna delle opere dovuto a problemi causati dalle vecchie fondamenta del Fafich, l'evento 50 anos de resistência à ditadura 1964 è stato realizzato nell'edifico prédio conosciuto come Coleginho, nell'antico complesso della Fafich, dove attualmente opera la Segreteria Municipale dell'Istruzione. Promossa dall'Associazione degli amici del Memoriale dell'Amnistia politica brasiliana, la cerimonia ha potuto contare sulla presenza di Jaime Arturo Ramírez e Sandra Goulart, rettore e vice-rettore della UFMG, di Eleonora Menicucci, Ministro della Segreteria delle politiche per le donne e Paulo Abrão, presidente della Commissione di Amnistia, nonché Segretario nazionale della Giustizia. Durante la cerimonia, venne diffusa la notizia che il Consiglio direttivo del Patrimonio culturale di Belo Horizonte si era riunito in via straordinaria per mettere sotto tutela l'edificio che «era stato uno dei luoghi principali della resistenza ai militari che presero il potere e destituirono João Goulart dalla Presidenza»4.

La messa sotto tutela dell'edificio, che sarebbe entrato a far parte del progetto del Memoriale dell'Amnistia, che circonda e fa parte dell'antico complesso della Facoltà di Filosofia e Scienze sociali, inserisce nella narrazione storica del patrimonio della città di Belo Horizonte la militanza e la resistenza politica del movimento studentesco e dell'intellettualità che componeva il suo personale accademico. Il provvedimento volto alla conservazione delle sue fondamenta e delle facciate moderne - di scarso valore estetico - reiterò la concezione di questo spazio come di un luogo della memoria della città dal punto di vista simbolico.

Pierre Nora ${ }^{5}$ rivela che i luoghi della memoria nascono dal senso di rottura con un passato dove il sentimento di frammentazione e disfacimento della memoria collettiva dà avvio a una vigilanza commemorativa intorno ai suoi luoghi. Attraverso lo spazio è possibile ancorare e cristallizzare queste memorie per ravvivare in maniera residuale la sensazione di continuità. Luogo di memoria della dittatura, il campus dell'antica Fafich, in quanto spazio scelto per edificare il Memoriale dell'Amnistia, è un luogo duplice:

Memória - come uno sforzo di adeguamento al Plano Nacional de Direitos Humanos 3 (2007), dove viene definita la politica pubblica di Diritto alla Memoria e alla Verità.

${ }^{3}$ MEZAROBBA, Glenda, Um acerto de contas com o futuro: a anistia e suas consequências um estudo do caso brasileiro, Tesi in Scienze politiche, Universidade de São Paulo, São Paulo, 2003 , p. 146.

${ }^{4}$ UFMG, «Evento na futura sede do Memorial da Anistia celebra resistência à ditadura», in Universidade Federal de Minas Gerais, URL: https://www.ufmg.br/online/arquivos/032606.shtml >, [consultato l'11 settembre 2015].

${ }^{5}$ NORA, Pierre, «Entre memória e história: a problemática dos lugares », in Projeto História, 10, 1993 pp. 7-29. 
«un luogo di eccesso», che ha fissato la sua identità nella lotta per la resistenza, «ma costantemente aperto ad un'estensione dei suoi significati» 6 .

Questo luogo della memoria della dittatura, in quanto locus di «affermazione della democrazia e dei diritti umani e di superamento dei traumi del passato» mira a promuovere il «ringiunzione dei brasiliani con la loro storia» attraverso una doppia funzione, pedagogica e morale. L'accesso ai documenti scritti, alle testimonianze, ai materiali audio e video raccolti all'interno di una narrazione di lotta resistenziale e di "riconquista della democrazia" disegna uno spazio di presa di coscienza, oltre ad ancorare nella realizzazione dell'edificio un mutamento di posizione dello Stato brasiliano attraverso la simbolica richiesta di perdono alle vittime e ai loro familiari che videro stroncate le loro vite o che furono barbaramente colpiti dalla persecuzione politica e dai crimini praticati dai regimi autoritari del nostro recente passato.

Storicamente il Brasile si è dimostrato un paese avvezzo alle amnistie. Sin dal periodo coloniale è possibile individuare differenti momenti ${ }^{7}$ in cui questi tentativi di "pacificazione dello spirito" furono utilizzati dai rappresentanti politici per liberare e ripristinare i diritti degli oppositori politici. Tuttavia l'ultima amnistia promulgata nel paese - Legge 6.683/1979 - permea la nostra memoria recente attraverso le immagini cristallizzate del tessuto sociale in cui un'ampia mobilitazione popolare invase lo spazio pubblico domandando un'amnistia "ampia, generale e senza restrizioni" e la commozione generata dalla liberazione dei prigionieri e dal rientro degli esiliati politici. Malgrado ciò il testo di legge approvato dal Congresso Nazionale, a cui un'ampia maggioranza aderì, lasciò fuori molte delle rivendicazioni e, soprattutto - nella sua interpretazione ambigua - promosse una "conciliazione" basata sull'oblio e sull'impossibilità di giudicare i militari responsabili di crimini contro l'umanità.

Secondo Paulo Abrão ${ }^{8}$, presidente della Commissione di Amnistia, a partire dal secondo decennio di questo secolo, è sorto un grande interrogativo riguardo alla

\footnotetext{
${ }^{6}$ Ibidem, p. 27.

${ }^{7}$ Secondo Mezarobba, decine di amnistie vennero concesse nel corso della storia brasiliana, nel periodo coloniale così come in quelli imperiale e repubblicano. Evidenziamo cronologicamente alcuni dei processi del concessione dell'amnistia che vengono citati nella sua dissertazione: nel 1654 la capitania di Pernambuco concesse l'amnistia ai nativi e ai portoghesi che collaborarono con l'occupazione olandese; nel 1822 Dom Pedro I, poco dopo l'indipendenza, concesse l'amnistia a tutte le persone - delle più disparate opinioni politiche -, eccetto che agli oppositori già in prigione o sotto processo; nel 1930 il governo provvisorio di Getúlio Vargas amnistiò tutti i civili e i militari che avevano partecipato, direttamente o indirettamente, ai movimenti rivoluzionari che si erano scatenati nel paese; nel 1945 Vargas concesse l'amnistia a tutti quelli che avevano commesso crimini politici a partire dal 15 luglio 1934, durante il periodo dell'Estado Novo. MEZAROBBA, Glenda, op. cit., pp. 2-5.

${ }^{8}$ ABRÃO, Paulo, TORELLY, Marcelo D., El programa de reparaciones como eje estructurador de la justicia transicional en Brasil, in RÉATEGUI, Félix (ed.), Justicia Transicional: manual
} 
giustizia di transizione in Brasile, incluso il tema dell'interpretazione giuridica che conferiva alla Legge di Amnistia un carattere "bilaterale". Nella storiografia è divenuta ricorrente la nozione dell'amnistia come un "chiudi la bocca" alle vittime, dal momento che la sua interpretazione "blindò" le possibilità di giudizio di coloro che si macchiarono di crimini all'interno del regime. Tuttavia, nell'attuale scenario transizionale, la costituzione di una Commissione di Verità (2012-2014) e di progetti di compensazione simbolica, come la costruzione del Memoriale dell'Amnistia, mostra che sono stati conseguiti progressi nella strutturazione di un più ampio programma di riparazione nei confronti delle vittime.

Per Abrão, l'idea di una domanda a posteriori di giustizia transazionale genera un'incognita nella letteratura specializzata, che molte volte non prende in considerazione il carattere strutturante del processo di compensazione. Per questa ragione la posizione della Commissione di Amnistia negli ultimi anni è stata quella di proporre una nuova lettura del processo transazionale brasiliano, che tenga conto della genesi e del processo di consolidamento dei diritti di compensazione, così come dello scenario opposto, quello dell'ampio controllo del regime dopo l'apertura politica del paese 9 .

Nelle ricerche più recenti, dove la lotta per la revisione dell'amnistia e dei suoi sviluppi è analizzata in quanto processo di lunga durata ${ }^{10}$ - scrutando il complesso quadro di eventi, personaggi e gruppi in costante disputa fra loro - comprendiamo come la problematica suscitata dall'eventuale responsabilizzazione di coloro che si sono macchiati di crimini rinfocoli nuovamente discussioni che oscillano tra la conciliazione attraverso l'oblio e la possibilità di consegnare alla giustizia i responsabili, con la motivazione che l'amnistia non comprende i crimini contro l'umanità come il sequestro, la tortura e l'occultamento di cadavere.

Questi temi continuano a restare oggetto di disputa e il Memoriale è ancora in costruzione. In questo articolo ci interessa mettere in evidenza come il progetto venga concepito a partire dall'idea di risignificazione del termine amnistia, legandola alla memoria della mobilitazione, della lotta e riferendola alla democrazia. Rodeghero ${ }^{11}$ afferma che i responsabili della campagna pro-amnistia e le misure che fecero seguito alla Legge del 1979 furono la risultante di giochi di forza: da una parte c'erano la

para América Latina, Brasília-Nueva York, Comisión de Amnistía delMinisterio de JusticiaCentro Internacional para la Justicia Transicional, 2011, pp. 477-521.

${ }^{9}$ Ibidem.

${ }^{10}$ MEZAROBBA, Glenda, op. cit.

${ }^{11}$ RODEGHERO, Carla Simone,Anistia, esquecimento, conciliação e reconciliação: tensões no tratamento da herança da ditadura no Brasil, in Marcas da Memória: história oral da anistia no Brasil, Recife, Ed, Universitária da UFPE, 2012, pp. 97-135. 
dimensione della lotta e la mobilitazione di importanti settori della società civile, dall'altro il tentativo di "transizione controllata" di Figueiredo, l'ultimo presidente militare (1979-1985). Nel 1975, diverse organizzazioni (come, tra gli altri, il Movimento Feminino pela Anistia, i Comitês Brasileiros de Anistia, le organizzazioni per la difesa dei diritti umani, o il partito politico Movimento Democrático Brasileiro e l’Ordine degli avvocati del Brasile) lottavano per la liberazione dei prigionieri politici e militari allontanati dal loro luogo di lavoro dal regime e per il chiarimento delle circostanze di decessi e sparizioni.

Nel 1978 e nel 1979 si intensificò la lotta per ottenere un'amnistia ampia, generale e senza restrizioni. In questo contesto, l'opposizione costruì un discorso sulla base del quale l'amnistia, oltre che ampia, generale e senza restrizioni, avrebbe dovuto essere reciproca, perché la pace sociale poteva essere instaurata solamente a partire dal mutuo perdono tra torturatori e prigionieri politici. La vittoria della proposta di "conciliazione per il rinnovamento" di Figueiredo fu in grado di assorbire elementi del discorso dell'opposizione per giustificare la sua proposta, ma mantenne fermo il proposito di continuità con il passato. Rodeghero ${ }^{12}$ sottolinea come dalla fine degli anni Settanta, tanto l'opposizione quanto il governo associarono l'amnistia all'idea della «riconciliazione della famiglia brasiliana», indicando una scelta fra le proposte che parevano più adeguate ad una logica di transizione sicura. Pertanto trasferendo il significato dell'amnistia in quanto strumento di conciliazione, in un dispositivo di riconciliazione orientato allo sforzo critico di tracciare un bilancio storico, si enfatizza l'attuale giustizia di transizione, con conseguenze dirette sulla concezione del Memoriale.

Una delle ragioni che riaffermano il proposito di revisione del passato finalizzato alla promozione della riconciliazione e dell'unità nazionale è esplicitata dalla stessa scelta del luogo di costruzione del Memoriale, Belo Horizonte.

Il memoriale sarà realizzato a Belo Horizonte, capitale dello stato di Minas Gerais, la cui bandiera e la cui storia esaltano la difesa della libertà. La sua sede entrerà in funzione nel quartiere di Santo Antônio, conosciuto per la sua intellettualità e per l'attività culturale intensa, in un edificio messo sotto tutela a fianco dell'antica Facoltà di Filosofia e Scienze umane (Fafich). Si tratta di un luogo riconosciuto per la gestazione delle idee, per il dibattito politico e per essere stato una cassa di risonanza di ogni comportamento di avanguardia dell'epoca.

${ }^{12}$ Ibidem. 
La scelta si deve anche al ruolo giocato dalla UFMG durante la dittatura militare e per la qualità delle sue ricerche sul periodo, svolte principalmente dal gruppo del Projeto República ${ }^{13}$.

Nel suo studio sulle rappresentazioni, Lefebvre accetta il rappresentativo come un fatto sociale, psichico e politico. Tenta di illustrare i percorsi per le forme di rifiuto globale di alcune rappresentazioni. Pensata come una costruzione, l'autore sostiene che attraverso l'opera (non solamente l'opera d'arte, ma qualsiasi opera umana ben fatta, come una città, o un'opera urbana o monumentale) sia possibile comprendere come le rappresentazioni la attraversino, dal momento che «la rappresentazione non consiste in un insieme di immagini, un riflesso o in una qualsiasi astrazione, se non in una mediazione» ${ }^{14}$. Sono queste mediazioni che attraversano il progetto di costruzione del Memoriale, sia per rifiutarlo che per legittimarlo, che ci interessano nella discussione proposta da questo articolo.

Nella sua materialità e monumentalità, il Memoriale ci rivela solamente la superficie delle sue rappresentazioni. Ciò che ci interessa è discutere il processo di costruzione simbolico che si rivela attraverso questo progetto politico. Perché Belo Horizonte è stata scelta come città per realizzare un luogo della memoria della dittatura di questa portata? Quale capitale politico viene mobilitato o risignificato in questo processo? Sono queste le domande a cui cercheremo di rispondere nel prossimo paragrafo.

\section{Miti fondatori della Repubblica: cospirazione, mineirismo e libertà}

Come abbiamo accennato nel paragrafo precedente, la costruzione del Memoriale nell'attuale scenario di giustizia transazionale mobilita alcuni valori legati ai miti e alle simbologie repubblicane. La "difesa della libertà" rappresentata dalla bandiera di Minais Gerais simboleggia l'ideale repubblicano fissato poco dopo la sua proclamazione attraverso la costruzione della narrazione di un mito fondatore - Tiradentes e la Inconfidência Mineira ${ }^{15}$ - e rinforzato dalla realizzazione di una città progettata per

13 PISTORI, Edson Claudio, SILVA FILHO, José Carlos Moreira da, «Memorial de Anistia Política sai do papel », in Revista Anistia Política e Justiça de Transição, 1, 1/2009, pp. 114-134, p. 116.

${ }^{14}$ LEFEBVRE, Henri, La présence et l'absence, Paris, Casterman, 1980, p. 37.

15 Tiradentes, pseudonimo di Joaquim José da Silva Xavier, diede vita - insieme ad altri congiurati - ad un tentativo di insurrezione, l'Inconfidência Mineira, il Tradimento mineiro, 
essere la sintesi delle idee positiviste di questo nuovo progetto politico, la Cidade de Minas, successivamente denominata Belo Horizonte.

La proclamazione della repubblica fu una questione politica, che vide una scarsa partecipazione sociale. L'intervento militare delle truppe del maresciallo Deodoro da Fonseca, primo presidente del Brasile (1889-1891), appoggiate da settori politici repubblicani, non mutarono immediatamente le condizioni delle popolazioni o delle città brasiliane. Le manifestazioni popolari, nelle loro diverse rivendicazioni furono soffocate dal potere. In considerazione delle crisi costanti e per legittimare il nuovo contesto e progetto politico era necessario ancorarlo saldamente e rappresentarlo attraverso immagini fondative, che promuovessero un sentimento di identificazione della popolazione con questi simboli e, conseguentemente, che creassero un'unità nazionale. Lo scarso radicamento popolare della repubblica nel paese rendeva imperativa la traduzione delle idee e delle aspirazioni del nuovo regime nella figura di un eroe che parlasse «alla testa e al cuore dei cittadini» ${ }^{16}$.

L'eroe nazionale eletto per rappresentare il panteon civico della Repubblica fu Joaquim José da Silva Xavier (1746-1792). Conosciuto come Tiradentes, fece parte del gruppo di cospiratori di Vila Rica, che complottarono per liberare le capitanie di Minas Gerais, Rio de Janeiro e di São Paulo dal dominio portoghese. Separati e catturati dalla corona portoghese, che vedeva nella cospirazione un tentativo di delegittimare il patto coloniale, furono processati e condannati. Tiradentes venne scelto per un'esecuzione esemplare sulla pubblica piazza: l’impiccagione seguita dalla squartamento del suo corpo.

Nel suo studio su Tiradentes come eroe della Repubblica, Carvalho ${ }^{17}$ giustifica la scelta di un personaggio storico del periodo coloniale a detrimento di personaggi coinvolti nella proclamazione della Repubblica alla fine del secolo XIX, come Ruy Barbosa, Floriano Peixoto e lo stesso maresciallo Deodoro.

La mancanza di un coinvolgimento reale del popolo nell'instaurazione del regime porta a un tentativo di compensazione, attraverso la mobilitazione simbolica. Ma, dal momento che la creazione di simboli non è arbitraria, non si fa nel vuoto sociale, ciò fa sì che si generino maggiori difficoltà per la realizzazione di un panteon civico. Herói que se preze tem de ter, de algum modo, a cara da nação. Devono rispondere

contro la corona portoghese che imponeva una forte tassazione. Tiradentes si assunse tutta la responsabilità dell'insurrezione, nell'intento - vano - di scagionare gli altri congiurati; fu condannato alla forca e, successivamente, il suo corpo venne squartato e la sua testa fu esposta a Vila Rica, l'antico nome di Ouro Preto [N.d.T.].

${ }^{16}$ CARVALHO, José Murilo de, A Formação das Almas: o imaginário da República no Brasil, São Paulo, Companhia das Letras, 1990, p. 55.

${ }^{17}$ Ibidem. 
ad alcune necessità o aspirazioni collettive, riflettere qualche tipo di personalità o di comportamento che corrisponda ad un modello valorizzato collettivamente ${ }^{18}$.

Carvalho sottolinea alcuni fattori importanti che giocarono a favore dellasua trasformazione in martire della Repubblica. A partire dalla prima metà del XIX secolo, la figura di Tiradentes già era venerata per il fatto che lo Stato era considerato geograficamente come il centro politico del paese e qui era «più forte il repubblicanesimo e più diffusi i clubs Tiradentes ${ }^{19}$. La sua figura agiva anche sul piano simbolico con una forte identificazione di stampo mistico ed etico. Senza tracce della sua reale fisionomia, questo martire senza volto venne perlopiù rappresentato nei dipinti facendo riferimento alla rappresentazione più comune e ricorrente di Gesù Cristo: capelli e barba lunga, una tunica bianca che lo circondava di un'aura mistica e un'espressione di perdono e compassione. Questo Tiradentes allegorico univa la società repubblicana intorno al sentimento di partecipazione e a un ideale che, nel corso della storia recente del XX secolo, venne utilizzato con le finalità più disparate, sempre in nome di una "libertà", pretesa dalle differenti colorazioni politiche.

La rilevanza di Tiradentes, fino al giorno d'oggi, è indiscutibile. Con la data della sua morte che venne decretata festività nazionale nel 1890, viene ricordato ogni anno il senso del sacrificio del suo sangue, versato per la libertà. Il suo nome viene attribuito a vie, piazze ed edifici, la sua immagine sconosciuta prende corpo in statue e monumenti in diverse città brasiliane. Dal momento che sono cambiate le rappresentazioni che associano valori a questo personaggio storico, comprendiamo anche come il progetto repubblicano della fine del XIX secolo finisse per glorificare il XVIII secolo come rappresentativo della tradizione e della lotta libertaria, "prodotta" da un nativo, in uno spazio di grande importanza geografica, politica ed economica durante la corsa all'oro.

Nello stesso tempo in cui il progetto repubblicano mobilitò l'ideale della libertà per creare le basi dell'agognata unità nazionale, consolidò nel suo patto federativo quell'apertura grazie alla quale gli stati federali avrebbero potuto legittimare il loro potere attraverso la costruzione di nuove capitali in grado di realizzare gli ideali positivisti di "ordine e progresso". L'utopia scientista della fine del XIX secolo cercava di abbandonare i residui del passato imperiale, proiettando nello spazio la sua prospettiva per il futuro. Nel Minas Gerais,per legittimare il nuovo assetto politico che si stabilì con l'instaurazione della Repubblica, divenne necessario abbandonare l'antica

\footnotetext{
${ }^{18}$ Ibidem.

${ }^{19}$ CARVALHO, José Murilo de, op. cit., p. 67.
} 
capitale di Ouro Preto che per l'esaurimento delle miniere e il disgregamento economico e sociale aveva finito per essere associata alla decadenza e all'inattività.

Con il decreto del 15 giugno 1891, la Costituzione politica dello Stato, a partire da due disposizioni transitorie stabilì lo spostamento della capitale in un luogo che fosse dotato delle condizioni igieniche necessarie per la costruzione di una grande $\operatorname{città}^{20}$. Questa nuova misura portò a una divisione all'interno dei gruppi politici tradizionali (sia del Centro, sia del Nord) e alla creazione di nuove egemonie legate alle zone di produzione del caffè (del Sud e della zona costiera), che talvolta ventilavano la possibilità di separarsi dal territorio mineiro.

Vincendo una debole resistenza dei politici di Ouro Preto, Belo Horizonte venne eletta come luogo ideale per progettare la nuova capitale. Se da un lato rappresentava il sogno del progetto positivista di futuro e modernità, grazie a una città pensata e pianificata nei termini di una pianificazione moderna e pragmatica,

il vero progetto del gruppo conservatore è spostare la capitale in un luogo più centrale dello Stato, con un futuro piano di sfruttamento minerario che possa risollevare l'economia. È una proposta conservatrice perché nasce in risposta ad una situazione di pericolo e non all'interno di un rilevante processo di mutamento economico. Tuttavia questa corrente ne esce vincitrice venendo inclusa nel nuovo gruppo come finanziatrice del progetto ${ }^{21}$.

Aarão Reis (1853-1936), ingegnere formatosi alla Escola Politécnica do Rio de Janeiro, venne scelto dal gruppo moderato dell'oligarchia dominante per studiare la località e, successivamente, per portare a termine la costruzione della nuova capitale. In sintonia con la filosofia positivista, razionalizzò la sua opera a partire da uno schema basato su tre grandi vie che si dipartivano dal cuore della capitale, la Praça da Liberdade. Attraverso queste diresse il sentimento di crescita e occupazione, nello stesso tempo in cui ancorava la rappresentazione politica del progetto nell'architettura degli edifici pubblici. La montagna della Serra do Curral diventò lo spazio del miraggio, in cui si poteva avvistare il paesaggio della modernità da consolidare nel futuro con «le locomotive in movimento, i pali del telegrafo, i viaggiatori in movimento e, soprattutto, l'architettura inusitata della stazione» ${ }^{22}$.

\footnotetext{
${ }^{20}$ ANDRADE, Rodrigo Ferreira, MAGALHÃES, Beatriz de Almeida, A Formação da Cidade, in CASTRIOTA, Leonardo Barci (org.), Arquitetura da modernidade, Belo Horizonte, UFMG, 1998, p. 41; ANGOTTI-SALGUEIRO, Heliana, «Da natureza ao construído», in Revista do Arquivo Público Mineiro, 43, 2/2007, pp. 44-59.

${ }_{21}^{21}$ ANDRADE, Rodrigo Ferreira, MAGALHÃES, Beatriz de Almeida, op. cit., p. 41

${ }^{22}$ ANGOTTI-SALGUEIRO, Heliana, op. cit., p. 51.
} 
La Commissione edilizia risultò fondamentale nella realizzazione di una narrativa della nuova capitale. La preoccupazione di Aarão Reis di documentare con testi e immagini tutte le tappe dell'evoluzione della costruzione città, oltre a costituire materiale di propaganda per il nuovo progetto, costituì anche un discorso fondativo per la città. Mentre la pianta della città - un tracciato geometrico, moderno e razionale era legato alle idee di natura, progresso e civilizzazione, la sua proposta politica mostrava un intento differente, conciliando forze modernizzatrici e conservatrici ${ }^{23}$. Belo Horizonte vide la posa della sua prima pietra nella Praça da Liberdade - "evocata" da Tiradentes - per incamminarsi assieme verso il nuovo progresso sognato dalla Prima Repubblica. Secondo Mello,

[...] l'atto di fondare Belo Horizonte permise di individuare in Ouro Preto le radici originali del Minas e, al contempo, di trasformarla in centro consacrato della Repubblica brasiliana. Spazio che avrebbe potuto aiutare a fissare e identificare la nazione brasiliana con lo Stato sotto l'egida della Repubblica. In questo modo, Belo Horizonte nacque come l'altra faccia della medaglia [rispetto a Belo Horizonte] ${ }^{24}$.

Con il passare del tempo possiamo notare come questo doppio progetto di trasformare Ouro Preto nella reliquia della nazione e Belo Horizonte nella città della speranza nel futuro venne legittimato a partire dalle politiche patrimoniali in atto per identificare, selezionare e preservare l'identità della nazione ${ }^{25}$. L'affermazione del discorso inerente al patrimonio relativamente a Ouro Preto, in quanto spazio sacro della nazione, ebbe inizio con viaggi esplorativi di importanti personalità che presero parte alla Settimana dell'Arte Moderna del 1922. Cercando di ordinare e riconoscere la vera identità nazionale, figure come Mário de Andrade, Oswald de Andrade e Tarsila do Amaral trovarono nel Minas la figura del sertanejo - l'abitante dell'entroterra - e, in particolare a Ouro Preto, l'architettura coloniale che riconobbero come il vero gioiello dell'arte brasiliana.

Durante l'Estado Novo (1937-1945), con la creazione dell'Istituto nazionale del Patrimonio storico e artistico brasiliano, il Serviço do Patrimônio Histórico e Artístico Brasileiro (SPHAN), importanti personalità mineiras iniziarono, all'interno

\footnotetext{
${ }^{23}$ ANGOTTI-SALGUEIRO, Heliana, op . cit.

${ }^{24}$ MELLO, Ciro Flávio Bandeira de, A noiva do trabalho - uma capital para a República, in DUTRA, Eliana de Freitas (org.), BH: horizontes históricos, Belo Horizonte, C/ARTE, 1996, pp. 11-48, p. 35 .

${ }^{25}$ BRAGA, Vanuza Moreira, Relíquia e esperança: Ouro Preto e as políticas de preservação do patrimônio no Brasil, in FERREIRA, Marieta de Moraes (org.), Memória e Identidade Nacional, Rio de Janeiro, Editora FGV, 2010, pp. 181-216.
} 
dell'istituzione, la costruzione di una narrazione dell'“autentico" patrimonio brasiliano, all'interno della quale la combinazione di arte religiosa barocca e lo stile coloniale delle casette ouropretane furono eletti come rappresentanti legittimi del beni culturali brasiliani. Ouro Preto come santuario di un panteon civile, messo sotto tutela e preservato dalle politiche pubbliche, fu consegnato simbolicamente alla nazione dal presidente Getúlio Vargas (1882-1954), quando decretò il ritorno delle spoglie di Tiradentes nella sua città natale e nella memoria di Ouro Preto. Con il passato della nazione fissato nell'antica capitale mineira, Belo Horizonte si trasformava ne «l'altro lato della medaglia» ${ }^{26}$.

La forza del tema "libertà", evocato dall'evento della Inconfidência, fu uno strumento imprescindibile per legittimare la base repubblicana. Il suo mantenimento venne promosso nel corso del XX secolo dall'amministrazione pubblica a partire dalle date commemorative, dalla retorica e dalla costruzione di monumenti quali modalità di ritualizzare gli "echi” dell'eroe nazionale. Impedendo che l'immagine di Tiradentes svanisse, più volte la sua mitologia venne evocata da personalità e gruppi politici. Animate dall'ambiguità, le azioni politiche che si appropriavano della sua allegoria repubblicana avevano più l'intento di reiterare il suo smembramento attraverso la manipolazione politica piuttosto che di rafforzare i valori positivisti della sua rappresentazione simbolica.

A Belo Horizonte, Faria ${ }^{27}$ segnala la presenza di due monumenti importanti per rinforzare l'idea di un passato comune della nazione: il Monumento alla Terra Mineira (1930) e la statua di Tiradentes (1962). Il primo, riaffermava il passato dei bandeirantes ${ }^{28}$ e degli Inconfidentes come fondativo dell'identità mineira, attraverso immagini scolpite su grandi placche di bronzo. Il secondo, dava le sembianze, attraverso linee moderne, a un busto del proto-martire Tiradentes. In entrambi i casi, «l'idealizzazione politica e morale degli Inconfidentes trasmessa durante i discorsi inaugurali ambiva non solamente alla perpetuazione di un esempio, ma alla creazione di un’immagine speculare rispetto a quelle amministrazioni»29.

Tiradentes e la memoria dell'Inconfidência non vennero utilizzati solamente con finalità “elettorali”. La stessa idea di conquista della libertà attraverso una cospirazione sarebbe stata associata ad un gruppo politico mineiro, che durante gli anni Sessanta si

\footnotetext{
${ }^{26}$ MELLO, Ciro Flávio Bandeira de, op. cit.

${ }^{27}$ FARIA, Carlos Aurélio Pimenta de, A memória cinzelada: em busca de uma consciência político-social - análise dos monumentos belorizontinos aos Inconfidentes, in DUTRA, Eliana de Freitas (org.), BH: horizontes históricos, Belo Horizonte, C/ARTE, 1996, pp. 289-332.

${ }^{28}$ Nota su

${ }^{29}$ FARIA, Carlos Aurélio Pimenta de, op. cit., p. 329.
} 
denominò Novos Inconfidentes. La formazione del gruppo fu frutto del legame politicoimprenditoriale, che culminò nel golpe civile-militare del 1964, attraverso un complesso accordo sostenuto dall'Instituto de Pesquisas e Estudos Sociais (IPES). L'IPES di Rio de Janeiro e quello di São Paulo agirono come una comunità politicoimprenditoriale per la destabilizzazione e la destituzione del presidente João Goulart (1961-1964). Le Reformas de base che marcarono il governo di Jango, come era soprannominato João Goulart, divennero sempre più impopolari per politici, imprenditori e militari, che avevano un proprio progetto politico-ideologico, che René Dreifuss $^{30}$ ha definito come un progetto di classe di interessi multinazionali associati, allineato con l'interesse nordamericano volta a espandere l'economia capitalista nell'America Latina.

Per realizzare il suo obiettivo strategico, «l'IPES avrebbe avuto efficacia reale solamente se si fosse rivelato capace di svolgere la sua azione politico ideologica in ambito nazionale» ${ }^{31}$. Nel progetto di espansione dell'IPES per le diverse regioni del paese, Minas Gerais diveniva fondamentale per l'influenza della sua élite politica sull'amministrazione federale. Anche altri fattori erano in gioco: il Minas delimitava anche la principale area di concentrazione industriale del paese e geograficamente era strategico per la resistenza militare. La posizione geografica centrale nel Brasile aumentava le sue possibilità di comunicazione e completava, assieme al centro nervoso (gli Stati di Rio de Janeiro e Saõ Paulo), il triangolo dell'articolazione del golpe. Circondata da una catena montuosa, Minas Gerais svolgeva una duplice funzione in quanto arena del possibile combattimento: «rifugio e punto di slancio delle forze militari» 32 .

L'IPES mineiro aveva intrapreso la grande sfida di creare una struttura unificata, in cui gli interessi dell'imprenditoria "tradizionale", del settore intermedio "moderno" e dei poteri bancari mineiros andassero di comune accordo. Fu con questo intento che il gruppo mobilitò rappresentazioni dell'Inconfidência Mineira in favore del proprio suo discorso legittimatore. Così come gli Inconfidentes del 1789, i Novos Inconfidentes di Belo Horizonte associarono il carattere cospirativo per giustificare l'assenza di partecipazione sociale delle classi subalterne al progetto di “insurrezione”. Rimuovendo i rischi di una rivolta popolare, i Novos Inconfidentes mantennero il loro progetto elitista, mentre si appropriavano del richiamo universale all'ideale di libertà.

\footnotetext{
${ }^{30}$ DREIFUSS, René Armand, 1964: A conquista do Estado, Petrópolis, Vozes, 1981.

${ }^{31}$ STARLING, Heloísa Maria Murgel, Os senhores das Gerais: os Novos Inconfidentes e o Golpe Militar de 1964, Petrópolis, Vozes, 1986, p. 48.

${ }^{32}$ Ibidem.
} 
Articolata al discorso politico-ideologico di parti della classe dominante, la "libertà benché tardiva" del 1789 venne utilizzata nel 1964, dall'IPES-“Novos Inconfidentes", come la grande bandiera ideologica dell'opposizione ai tentativi di "golpe comunista nel paese" che le élites conservatrici tanto temevano. Si trattava, sì, della libertà, ma per come le élites mineiras la comprendevano e la accettavano: la libertà che si doveva imporre33.

Svincolata dal suo momento storico originale, la cospirazione dei Novos Inconfidentes negli anni Sessanta se ripeté in farsa ${ }^{34}$. L'appropriazione della rappresentazione dell'evento del 1789 si sviluppa sul piano simbolico come "resistenza all'oppressione in generale" ed è «radicata al "vivere a Minas" all'essere mineiro"». In questo senso è la stessa ambiguità che c'è intorno all'immagine di Tiradentes, che permette la sua vitalità in quanto eroe nazionale e, allo stesso modo, attraverso i «continui tentativi di smembramento della sua memoria»35, ha risonanza nell'immaginario sociale.

\section{Considerazioni finali}

L'instaurazione della Repubblica in Brasile marcò un periodo di rottura con il passato imperiale. Il progetto positivista intrapreso dall'élite politica brasiliana, in un gioco di forze per promuovere la stabilità e scongiurare il timore delle trasformazioni in corso, gettò le fondamenta della sua narrazione nazionale per conciliare la società brasiliana. Attraverso un ingegnoso processo di costruzione di significati, Tiradentes fu eletto eroe della nozione per "liberarci" dai legami con il Portogallo e permetterci di guardare al futuro, al nuovo.

I segni dell'identità nazionale furono abilmente mobilitati per equilibrare le domande di "tradizione" e di "modernità". Come abbiamo visto nel corso di questo articolo, la vigilanza commemorativa intorno all'episodio dell'Inconfidência Mineira permise che i suoi valori fossero utili a giustificare i più contraddittori esperimenti politici: una città progettata a tavolino e trapiantata ai piedi dell'antico Curral Del Rei, monumenti in bronzo dedicati a Tiradentes e all'Inconfidência in quel di Belo

\footnotetext{
${ }^{33}$ STARLING, Heloísa Maria Murgel, Os senhores das Gerais: os Novos Inconfidentes e o Golpe Militar de 1964, Petrópolis, Vozes, 1986, p. 86.

${ }^{34}$ Karl Marx, analizzando il golpe che portò Napoleone III al potere, concluse che l'azione era una ripetizione del golpe conosciuto come il colpo di stato del 18 brumaio realizzato da suo zio Napoleone Bonaparte. In quest'opera il filosofo coniò la celebre frase: «la storia si ripete, la prima volta come tragedia, la seconda come farsa».

${ }^{35}$ CARVALHO, José Murilo de, A Formação das Almas: o imaginário da República no Brasil, São Paulo, Companhia das Letras, 1990, p. 73.
} 
Horizonte per promuovere il personalismo politico e la preservazione di uno stile architettonico barocco-coloniale per narrare l'“autenticità" della nazionalità brasiliana.

Nel suo studio sulla retorica del patrimonio culturale in Brasile, Gonçalves ${ }^{36}$ afferma che le narrazioni dell'identità e del passato nazionale vengono promosse per dare coerenza e unità a un'idea reificata di questa entità che chiamiamo nazione. L'autore ci mette sull'avviso circa il fatto che questi discorsi, costruiti dal potere politico, sono processi illusori, dal momento che consistono «nel tentativo di superare o trascendere questa distanza o mancanza attraverso mezzi narrativi»37. Il compito è senza fine dal momento che le narrazioni nazionali sono sempre in discussione.

In questo processo, quella distanza o assenza, la distanza tra linguaggio ed esperienza, tra simbolo e ciò che è simboleggiato, significante e significato, desiderio e oggetto del desiderio, viene permanente ricreata benché sotto l'impulso magico di trascenderla ${ }^{3}$.

Nell'attuale dovere della memoria di rileggere il passato della dittatura civile militare (1964-1985), riteniamo che il Memoriale dell'Amnistia in quanto patrimonio miri a inscrivere nella memoria ufficiale della nazione la voce delle vittime del periodo - ex prigionieri politici, morti, desaparecidos e familiari delle vittime - attraverso una narrazione il cui filo conduttore è la lotta per la resistenza e il mantenimento della democrazia. Scegliendo Belo Horizonte come spazio fisico e simbolico per la costruzione del Memoriale, lo Stato brasiliano trova la possibilità di mobilitare l’ideale di "libertà", in opposizione alle nozioni mobilitate dai Novos Inconfidentes e all'uso indiscriminato della figura di Tiradentes nella promozione della coesione interna dello Stato castrense.

L'impulso che guida le politiche patrimoniali nell'attuale scenario di giustizia transazionale mostra un «segnale di rottura [...] tra un presente e un passato» 39 che ancora cerca unità narrativa e pacificazione sociale. La riconciliazione attraverso l'amnistia, in quanto memoria della lotta per il ritorno della democrazia e garanzia dell'ordinamento repubblicano, prevede di dipanare il suo filo narrativo a partire dalla città delle libertà. Nel suo ruolo di città-memoria della costruzione della narrazione della nazione, Belo Horizonte accoglie nei suoi archivi pubblici, monumenti e musei, la

${ }^{36}$ GONÇALVES, José Reginaldo Santos, A retórica da perda: os discursos do patrimônio cultural no Brasil, Rio de Janeiro, Editora UFRJ/IPHAN, 1996.

${ }^{37}$ Ibidem, p. 26.

${ }^{38}$ Ibidem, p. 21.

${ }^{39}$ HARTOG, François, «Tempo e patrimônio», in Varia historia, 22, 36, 2/2006, pp. 261-273, p. 272. 
memoria dei dirigenti e dei gruppi politici che pensarono e progettarono la nazione. Progetti imposti e analizzati a porte chiuse, reiterando e risignificando l'ethos cospirativo mineiro.

La capacità del progetto del Memoriale dell'Amnistia nel mobilitare questi valori repubblicani di libertà non ci sembra, in un primo momento, un'operazione ingenua 0 casuale. Con i processi della Commissione di Amnistia del Ministero della Giustizia e le testimonianze degli amnistiati, salvaguardati da un'istituzione di forte tradizione accademica come l'UFMG, si crea un luogo di memoria oltre a ribadire il concetto del dovere di ricordare affinché non si ripetano più le violazioni perpetrate nel passato recente. Negli intenti dei ricercatori del Projeto República, che sta analizzando la realtà politica mineira, questo luogo di conservazione e ricordo servirà anche come un nuovo orizzonte per la produzione di conoscenza e di nuove narrazioni del regime militare, oggi raccontato attraverso gli sguardi di coloro che vi furono addentro. 


\section{* L'autore}

Priscila Cabral Almeida, dopo avere conseguito la laurea in Memória Social presso il PPGMS dell'UNIRIO è attualmente dottoranda in História, Política e Bens Culturais del Centro de Pesquisa e Documentação de História Contemporânea do Brasil (CPDOC). Nel 2015 ha preso parte, come consulente al "Projeto de Cooperação Técnica BRA /o8/o21 - Cooperação para o intercâmbio internacional, desenvolvimento e ampliação das politicas de Justiç,a Transicional do Brasil”, stipulato tra il Programma delle Nazioni Unite per lo sviluppo (UNDP) e la Comissão de Anistia del Ministero della Giustizia brasiliano, che ha come obiettivo la creazione di processi di conservazione della memoria degli spazi e degli avvenimenti legati alla repressione politica e alla lotta resistenziale nello Stato di Bahia.

URL: < http://www.studistorici.com/progett/autori/\#CabralAlmeida >

\section{Per citare questo articolo:}

CABRAL ALMEIDA, Priscila, «Frammenti di dolore: violenza razziale-etnica nel cinema brasiliano durante la fase finale della dittatura militare (1979-1985)», Diacronie. Studi di Storia Contemporanea : Le dittature militari: fisionomia ed eredità politica, 29/12/2015,

URL:< http://www.studistorici.com/2015/12/29/cabral-almeida_numero_24/ >

Diacronie Studi di Storia Contemporanea $\vartheta$ www.diacronie.it

Risorsa digitale indipendente a carattere storiografico. Uscita trimestrale.

redazione.diacronie@hotmail.it

Comitato di redazione: Jacopo Bassi - Luca Bufarale - Elisa Grandi - Antonio César Moreno Cantano - Deborah Paci - Fausto Pietrancosta - Alessandro Salvador - Matteo Tomasoni - Luca Zuccolo

Diritti: gli articoli di Diacronie. Studi di Storia Contemporanea sono pubblicati sotto licenza Creative Commons 3.0. Possono essere riprodotti e modificati a patto di indicare eventuali modifiche dei contenuti, di riconoscere la paternità dell'opera e di condividerla allo stesso modo. La citazione di estratti è comunque sempre autorizzata, nei limiti previsti dalla legge. 